\title{
Vimentin and Ki67 expression in circulating tumour cells derived from castrate-resistant prostate cancer
}

\author{
C. R. Lindsay ${ }^{1 \dagger}$, S. Le Moulec ${ }^{2 \dagger}$, F. Billiot ${ }^{1}$, Y. Loriot ${ }^{3}$, M. Ngo-Camus ${ }^{3}$, P. Vielh ${ }^{1,4}$, K. Fizazi $^{3}$, C. Massard $^{3 \dagger}$ and F. Farace $^{1 *+}$
}

\begin{abstract}
Background: High circulating tumor cell (CTC) counts are associated with poor prognosis in advanced prostate cancer, and recently CTC number was suggested to be a surrogate for survival in metastatic castrate-resistant prostate cancer (mCRPC). Ki67 and vimentin are well-characterised markers of tumour cell proliferation and the epithelial-mesenchymal transition (EMT), respectively. Here we asked if the expression of vimentin and Ki67 in CTCs offered prognostic or predictive information in MCRPC.
\end{abstract}

Methods: In two separate patient cohorts, anti-vimentin or anti-Ki67 antibodies were added to the free channel in the CellSearch ${ }^{\oplus}$ system for analysis of peripheral blood samples. For each cohort, association of CTC number with clinical characteristics were assessed using Fisher's exact, Mann-Whitney and chi-squared tests. Kaplan-Meier method and log-rank tests were used to analyse overall survival (OS) of vimentin-expressing and Ki67-expressing CTC patient cohorts.

Results: In this retrospective analysis, CTC vimentin expression was analysed in 142 blood samples from 93 patients, and CTC Ki67 expression was analysed in 90 blood samples from 51 patients. In the vimentin cohort, 80/93 (86 \%) of baseline samples from patients were CTC-positive overall ( $\geq 1$ total CTC per $7.5 \mathrm{mls}$ blood), and 30/93 (32.3\%) vimentin CTC-positive ( $\geq 1$ vimentin-positive CTC per $7.5 \mathrm{mls}$ blood). 41/51 (80.4\%) of baseline samples from patients in the Ki67 cohort were CTC-positive overall, and 23/51 (45.1\%) Ki67 CTC-positive ( $\geq 1$ Ki67-positive CTC per $7.5 \mathrm{mls}$ blood). There was no significant difference in baseline PSA in patients with vimentin-positive CTC at baseline versus those with no vimentin-positive CTC at baseline $(p=0.33)$. A significant reduction in OS was shown in patients with vimentin-positive CTC compared to those without vimentin-positive CTC (median 305 days vs 453 days, $p=0.0293$ ). There was no significant difference in baseline PSA in patients with Ki67-positive CTC at baseline versus those without Ki67-positive CTC $(p=0.228)$, but OS was significantly reduced in the Ki67-positive CTC group (median 512 days vs 751 days, $p=0.0091$ ). No changes in relative proportion of vimentin- or Ki67positive CTCs were observed in post-treatment samples compared to baseline.

Conclusions: Analysis of vimentin and Ki67 expression can straightforwardly be assessed in CTCs from patients with mCRPC. Poorer survival outcomes were observed in vimentin- and Ki67-positive CTC patients.

Translational study protocols: CEC-CTC (IDRCB2008-AO0585-50) and Petrus (NCT01786031).

Keywords: Prostate, Vimentin, Ki67, Circulating

\footnotetext{
* Correspondence: francoise.farace@gustaveroussy.fr

†Equal contributors

${ }^{1}$ INSERM U981, University of Paris-Sud XI, Translational Research Laboratory,

Gustave Roussy Cancer Campus, Villejuif, France

Full list of author information is available at the end of the article
} 


\section{Background}

Circulating tumor cells (CTCs), captured as a 'liquid biopsy' from blood for enumeration and biological characterization of cancers, have the potential to replace biopsy and provide important clinical information on prognosis, therapeutic choice, and drug resistance, while also being of interest for drug development and biomarker discovery. They may represent an alternative source of tumor tissue which is easily accessible using a simple blood test, allowing longitudinal monitoring of tumor aggression and biology at different timepoints to guide therapeutic decisions in a patient's treatment course [1-3]. Prostate cancer was one of the first malignancies where the prognostic value of monitoring CTC numbers was demonstrated in patients with advanced disease, both before and during treatment for castrate-resistant prostate cancer (CRPC) using the FDA-approved CellSearch technology $[4,5]$. Moreover, a recent prospective trial demonstrated that CTC count and LDH value could be a surrogate of overall survival in a population of mCRPC patients treated with abiraterone in the COU-301 trial [6]. The potential of CTC for molecular characterization has been demonstrated on a number of occasions, most recently in CRPC with the demonstration of their variable androgen receptor (AR) expression [7-12].

$\mathrm{Ki}-67$ is a nuclear protein that is associated with ribosomal RNA synthesis and may be necessary for cell cycle proliferation. Its tissue staining has consistently demonstrated prognostic value in prostate cancer, and has been tested in men managed with radiation and surgery, as well as in those conservatively managed without definitive therapy [13-16]. One group of studies used pretreatment biopsies of patients undergoing radiation and androgen deprivation as part of the Radiation Therapy Oncology Group (RTOG) 92-02 trial, defining a cut-off of $11.3 \%$ high Ki-67 staining as independently correlated with an increase risk in distant metastasis, cancer-specific death, and overall death [13, 14]. A Mayo clinic study described similar outcomes from patient tissue taken before or during definitive prostatectomy, using a staining threshold of $6 \%$ high Ki67 (present in $11 \%$ of patients) which correlated with increased risks of cancer progression and cancer-specific mortality [16]. The phase III GETUG 12 study, which assessed androgen-deprivation therapy in high risk localized prostate cancer, showed that high Ki67 predicted an unfavourable PFS using a median Ki67 cutoff of $1 \%$ [17]. Finally, 243 conservatively-treated patients from the Transatlantic Prostate Group were shown to have significantly increased risk of cancer-specific mortality in the $5 \%$ of patients who had $>10 \% \mathrm{Ki} 67$ staining [15]. The detection of Ki67 in CTCs has been previously reported using a microfluidic CTC detection method, offering the opportunity to assess if longitudinal quantification of proliferative and non-proliferative subpopulations may be more clinically informative than examination of total CTC count and/or biopsy alone [18].

Vimentin is a filamental protein expressed in mesenchymal cells, often recorded as a marker of tumour cell invasion via its expression during activation of the epithelial-mesenchymal transition (EMT) [19, 20]. Compared to Ki67, its use in biological interrogation of CTC is more commonplace. Interest in EMT/vimentin in the CTC field continues due to the continuing biological challenge it represents: CTC undergo EMT in order to enter the bloodstream where they are captured, yet many current enrichment methods and definitions of CTC do not incorporate mesenchymal markers such as vimentin. It is hypothesized that, in cancers such as non-small cell lung cancer which have a low yield of CTC via EPCAM (epithelial)-antibody enrichment in CellSearch, many 'EMT' and mesenchymal CTC are missed as a consequence of sub-optimal enrichment methods [21-24]. Despite this potential problem, the validity of EMT analysis using the CellSearch platform has previously been confirmed by a report documenting the co-expression of vimentin with cytokeratin in samples from 10/10 CRPC patients, with 108/126 CTCs in total co-expressing these markers [25].

Given the association of Ki67 and vimentin with poor prognosis in metastatic CRPC biopsy, we hypothesized that vimentin or Ki67 expression in CTC would offer a poorer prognostic picture compared to that observed in patients with no vimentin- or Ki67-positive CTCs. We analysed Ki67 and vimentin expression in CTC derived from a total of 144 advanced prostate cancer patients using CellSearch technology, optimized and validated to assay Ki67 and vimentin in separate patient cohorts. Our results corroborate those seen in tumour biopsies, with a diminished OS observed in patients with vimentinexpressing CTCs and those with Ki67-expressing CTCs.

\section{Methods \\ Patients}

Peripheral blood $(7.5 \mathrm{ml})$ was collected from metastatic CRPC patients recruited to one of two GustaveRoussy CTC translational study protocols, CEC-CTC (IDRCB2008-AOO585-50) and Petrus (NCT01786031). For eligibility, patients had to have a confirmed diagnosis of metastatic CRPC (either by contemporaneous or historical biopsy). All patients offered their written informed consent and were enrolled on institutional protocols approved by the local Gustave-Roussy ethics review committee. The following minimal clinical data were collected: CTC count at baseline and following their first cycle of treatment (at cycles 2-3), date of CTC sample, date of progression or death (if applicable), histology, metastatic sites, systemic treatment due at time of baseline CTC sampling, and previous treatment. Routine laboratory 
analyses were also performed on patients at baseline and at first blood sample after initiation of treatment, including prostate-specific antigen (PSA) for response assessment. Patients were recruited for the analysis between December 2010 until November 2014.

\section{CellSearch}

Collection of blood and immunofluorescent (IF) staining of CTC was performed using the CellSearch ${ }^{\circ}$ system (Janssen Diagnostics, LLC), as previously reported $[4,26]$. Candidate cells (CTC +/- vimentin, or CTC +/- Ki67 all cytokeratin positive) were identified using the CellTracks Analyzer II (Janssen Diagnostics, LLC) according to methods and IF expression criteria previously reported [27]. The number of CTCs is presented per $7.5 \mathrm{ml}$ of blood. Unless otherwise stated in the text, patients in this study were defined as Ki67- or vimentin-CTC positive if they had $\geq 1$ vimentin-positive or Ki67-positive CTCs. For Additional file 1: Figure S3, two separate patient cohorts were assessed in exploratory analyses: for (a) and (c), patients with $\geq 5$ total CTCs and $\geq 1$ vimentin-positive or Ki67-positive CTC were compared with those patients with $\geq 5$ total CTCs and no vimentin-positive or Ki67positive CTC; for (b) and (d), the 10 CTC-positive patients with the highest proportions of vimentin/ Ki67-positive CTCs were compared with the 10 CTCpositive patients with the lowest proportions of vimentin/Ki67-positive CTCs.

\section{Ki67 and vimentin validation}

This was established using the CellSearch platform: in separate experiments, FITC- labelled anti-vimentin antibody (Santa-Cruz, 'vimentin cohort') and anti-Ki67 antibody (BD Biosciences, 'Ki67 cohort') were added to the free channel in the CellSearch system. Antivimentin was tested using donor blood spiked with A549 (vimentin + ve) and T47D (vimentin -ve) cancer cell lines (Additional file 1: Figure S1a). Anti-Ki67 was tested in donor blood spiked with A549 and SKBR3 cancer cell lines: in these cell lines, phytohaemagglutinin A was used to stimulate proliferation and anti-IgG was used as a negative antibody control (Additional file 1: Figure S1b). CellSearch runs were carried out at three different exposure $(0.4 \mathrm{~s}, 0.6 \mathrm{~s}, 0.8 \mathrm{~s})$ with no difference evident, thus $0.8 \mathrm{~s}$ was chosen as standard for analysis (data not shown). Experiments were repeated in duplicate.

\section{Statistics}

The association of CTC number with clinical characteristics and patient demographics were assessed using Fisher's exact test for dichotomous factors, and Mann-Whitney tests for continuous data. The Kaplan-Meier method was used to estimate overall survival, and the difference between CTC cohorts compared using the log-rank test. Chi-square tests were used to analyse categorical data. Data cutoff for survival estimates was March 1st, 2015: outcomes were censored if a patient had not died before this date. Overall survival is defined as the time from first CTC sample until death from any cause, cancer-related or otherwise. Statistics were analysed using GraphPad Prism 6.03 for Windows. All p values were two sided and considered statistically significant at $<0.05$.

\section{Results}

\section{Patient characteristics}

As only one free channel position for either Ki67 or vimentin antibodies was available using CellSearch, analyses were conducted in separate patient cohorts: a 'vimentin cohort' and a 'Ki67 cohort'. Patient details from each are recorded in Tables 1 and 2. From the vimentin cohort, 93 patients in total were recruited: 63 patients were vimentin CTC-negative (no vimentinpositive CTCs) at baseline and 30 patients were vimentin CTC-positive ( $\geq 1$ vimentin-positive CTC) (Tables 1 and 3, Fig. 1a). Both vimentin patient cohorts had similar baseline characteristics, other than an increase in visceral/multiple metastatic sites for patients in the vimentin CTC-positive group (43.3 vs $30.2 \%)$ which was consistent with more patients in this group reported to be receiving at least a 3rd line of systemic treatment ( 40 vs $22.2 \%$ ) (Table 1 ). The majority across both groups were post-docetaxel and due to receive at least $2^{\text {nd }}$ line systemic treatment (Table 1).

From the Ki67 cohort, 51 patients in total were recruited: 28 patients were Ki67 CTC-negative (no Ki67positive CTCs) and 23 patients were Ki67 CTC-positive at baseline ( $\geq 1$ Ki67-positive CTCs) (Tables 2 and 3, Fig. 1b). The majority of patients across the cohort were beyond 1st line systemic treatment. For the Ki67 CTCpositive group, increases in patients with $\geq 3$ metastatic sites (30.4 vs $14.3 \%$ ) and visceral metastatic sites (43.5 vs $21.4 \%$ ) were again consistent with more patients in this group reported to be receiving at least a 3rd line of systemic treatment (30.4 vs $10.7 \%$ ) (Table 2).

\section{CTC characteristics}

Table 3 provides a summary of CTC characteristics at baseline within the 2 cohorts. Vimentin expression was analysed in 142 samples from 93 patients: all patients were sampled at baseline, 49 of whom were also sampled at later timepoints during the same treatment regimen. 80/93 (86 \%) of baseline samples from patients were CTC-positive overall, and 30/93 (32.3\%) vimentin CTCpositive. $69 / 93$ (74.2 \%) patients belonged to the previously described 'unfavourable' prognostic category $(\geq 5$ standard CTCs) for overall CTC count, while 10/93 
Table 1 Baseline characteristics of patients with prostate cancer according to Vimentin CTC status

\begin{tabular}{|c|c|c|}
\hline Characteristic & $\begin{array}{l}\text { Vimentin CTC- } \\
\text { negative patients } \\
\text { (no vimentin + } \\
\text { ve CTCs) (\%) }\end{array}$ & $\begin{array}{l}\text { Vimentin CTC- } \\
\text { positive patients } \\
(\geq 1 \text { vimentin + } \\
\text { ve } C T C)(\%)\end{array}$ \\
\hline Total patient numbers & 63 & 30 \\
\hline \multicolumn{3}{|l|}{ Age, years } \\
\hline Median & 76 & 72 \\
\hline Range & $48-93$ & $53-86$ \\
\hline \multicolumn{3}{|l|}{ Number of metastasic sites } \\
\hline 1 & 26/63 (41.3) & $11 / 30(36.7)$ \\
\hline 2 & 21/63 (33.3) & $9 / 30(30)$ \\
\hline $3+$ & 15/63 (23.8) & $10 / 30(33.3)$ \\
\hline Not stated & 1/63 (1.6) & $0 / 30(0)$ \\
\hline \multicolumn{3}{|l|}{ Metastasic sites } \\
\hline Lymph Nodes alone & $3 / 63(4.8)$ & $1 / 30(3.3)$ \\
\hline Bone alone & $24 / 63(38.1)$ & 10/30 (33.3) \\
\hline Bone and lymph nodes & $17 / 63(27)$ & $6 / 30(20)$ \\
\hline Visceral/multiple sites & 19/63 (30.2) & $13 / 30(43.3)$ \\
\hline \multicolumn{3}{|c|}{ Treatment at time of $1^{\text {st }} \mathrm{CTC}$ specimen } \\
\hline Cabazitaxel & $7 / 63(11.1)$ & $7 / 30(23.3)$ \\
\hline Docetaxel & $7 / 63(11.1)$ & $3 / 30(10)$ \\
\hline Abiraterone & 26/63 (41.3) & $11 / 30(36.7)$ \\
\hline Enzalutamide & $5 / 63(7.9)$ & $2 / 30(6.7)$ \\
\hline Radium 223 & 2/63 (3.2) & $2 / 30(6.7)$ \\
\hline Others/Not Stated & $16 / 63(25.4)$ & $5 / 30(16.7)$ \\
\hline \multicolumn{3}{|c|}{ Line of treatment at time of $1^{\text {st }}$ CTC specimen } \\
\hline 1 & 10/63 (15.9) & $4 / 30(13.3)$ \\
\hline 2 & $17 / 63(27)$ & $8 / 30(26.7)$ \\
\hline $3+$ & $14 / 63(22.2)$ & $12 / 30(40)$ \\
\hline Not stated & 22/63 (34.9) & $6 / 30(20)$ \\
\hline \multicolumn{3}{|l|}{ Previous treatments } \\
\hline Primary surgery & 10/63 (15.9) & $7 / 30(23.3)$ \\
\hline Primary radiotherapy & $27 / 63(42.9)$ & $12 / 30(40)$ \\
\hline Docetaxel & $42 / 63(66.7)$ & $23 / 30(76.7)$ \\
\hline Enzalutamide & 2/63 (3.2) & $4 / 30(13.3)$ \\
\hline Cabazitaxel & 10/63 (15.9) & $7 / 30(23.3)$ \\
\hline Abiraterone & 21/63 (33.3) & $12 / 30(40)$ \\
\hline \multicolumn{3}{|l|}{ Total CTC levels at baseline } \\
\hline Mean & 98.8 & 492.6 \\
\hline Range & $0-1185$ & $1-4310$ \\
\hline
\end{tabular}

(10.8\%) patients had samples with $\geq 5$ vimentin-positive CTCs [4]. In the unfavourable prognostic category, vimentin-positive CTC prevalence was higher (28/69 samples, $40.6 \%)$. Only $1 / 93$ patients (1.1\%) had samples containing vimentin-positive CTCs exclusively, with the
Table 2 Baseline characteristics of patients with prostate cancer according to Ki67 CTC status

\begin{tabular}{lll}
\hline Characteristic & $\begin{array}{l}\text { Ki67 CTC-negative } \\
\text { patients (no Ki67 } \\
+ \text { ve CTCS) }(\%)\end{array}$ & $\begin{array}{l}\text { Ki67 CTC-positive } \\
\text { patients }(\geq 1 \text { Ki67 } \\
+ \text { ve CTC) (\%) }\end{array}$ \\
\hline $\begin{array}{l}\text { Total patient numbers } \\
\text { Age, years }\end{array}$ & 28 & 23 \\
Median & & \\
Range & 79.5 & 72 \\
\hline
\end{tabular}

Number of metastasic sites

$\begin{array}{lll}1 & 12 / 28(42.9) & 8 / 23(34.8) \\ 2 & 10 / 28(35.7) & 8 / 23(34.8) \\ 3+ & 4 / 28(14.3) & 7 / 23(30.4) \\ \text { Not stated } & 2 / 28(7.1) & 0 / 23(0) \\ \text { Metastasic sites } & & \\ \text { Lymph Nodes alone } & 0 / 28(0) & 0 / 23(0) \\ \text { Bone alone } & 12 / 28(42.9) & 8 / 23(34.8) \\ \text { Bone and lymph nodes } & 8 / 28(28.6) & 5 / 23(21.7) \\ \text { Visceral/multiple sites } & 6 / 28(21.4) & 10 / 23(43.5) \\ \text { Unknown/Not stated } & 2 / 28(7.1) & 0 / 23(0)\end{array}$

Treatment at time of $1^{\text {st }} \mathrm{CTC}$ specimen

$\begin{array}{lll}\text { Cabazitaxel } & 0 / 28(0) & 6 / 23(23.1) \\ \text { Docetaxel } & 6 / 28(21.4) & 3 / 23(13) \\ \text { Abiraterone } & 13 / 28(46.4) & 11 / 23(47.8) \\ \text { Others/Not stated } & 9 / 28(32.1) & 3 / 23(13)\end{array}$

Line of treatment at time of $1^{\text {st }} \mathrm{CTC}$ specimen

$\begin{array}{lll}1 & 5 / 28(17.9) & 3 / 23(13) \\ 2 & 8 / 28(28.6) & 5 / 23(21.7) \\ 3+ & 3 / 28(10.7) & 7 / 23(30.4) \\ \text { Not stated } & 12 / 28(42.9) & 8 / 23(34.8) \\ \text { Previous treatments } & & \\ \text { Primary surgery } & 5 / 28(17.9) & 3 / 23(13) \\ \text { Primary radiotherapy } & 6 / 28(21.4) & 5 / 23(21.7) \\ \text { Docetaxel } & 21 / 28(75) & 20 / 23(87) \\ \text { Enzalutamide } & 1 / 28(3.6) & 0 / 23(0) \\ \text { Cabazitaxel } & 1 / 28(3.6) & 0 / 23(0) \\ \text { Abiraterone } & 2 / 28(7.1) & 5 / 23(21.7) \\ \text { otal CTC levels at baseline } & & \\ \text { Mean } & 12.9 & 2-316 \\ \text { Range } & 0-79 & \end{array}$

other 29/30 vimentin-positive CTC samples harbouring at least one vimentin-negative CTC. 960/26299 (3.7 \%) of CTCs were vimentin-positive across baseline samples from the cohort.

For Ki67 expression, 90 samples from 51 patients were analysed: all patients were sampled at baseline, 39 of whom were also sampled at later timepoints during the 
Table 3 Baseline CTC characteristics of patients with prostate cancer according to vimentin and Ki67 CTC status

\begin{tabular}{ll}
\hline Vimentin cohort & \\
\hline Number of pts with $\geq 1$ CTC (\%) & $80 / 93(86)$ \\
Total CTC & $30 / 93(32.3)$ \\
$\quad$ Vimentin CTC & \\
Number of pts with $\geq 5$ CTC (\%) & $69 / 93(74.2)$ \\
Total CTC & $10 / 93(10.8)$ \\
$\quad$ Vimentin CTC & $28 / 69(40.6)$ \\
Number of pts with $\geq 5$ CTC and $\geq 1$ vimentin CTC & $1 / 93(1.1)$ \\
Number of pts with only Vimentin-positive CTC & $960 / 26299(3.7)$ \\
Number of Vimentin-positive CTCs in total & \\
Ki67 cohort & \\
Number of pts with $\geq 1$ CTC (\%) & $41 / 51(80.4)$ \\
Total CTC & $23 / 51(45.1)$ \\
Ki67 CTC & \\
Number of pts with $\geq 5$ CTC (\%) & $35 / 51(68.6)$ \\
Total CTC & $12 / 51(23.5)$ \\
Ki67 CTC & $22 / 35(62.9)$ \\
Number of pts with $\geq 5$ CTC and $\geq 1$ Ki67 CTC & $3 / 51(5.9)$ \\
Number of pts with only Ki67-positive CTC & $307 / 2495(12.3)$ \\
Number of Ki67-positive CTCs in total & \\
\hline
\end{tabular}

same treatment regimen. 41/51 (80.4\%) of baseline samples from patients were CTC-positive overall, and 23/51 (45.1 \%) Ki67 CTC-positive. 35/51 (68.6 \%) patients belonged to the 'unfavourable' prognostic category $(\geq 5$ standard CTCs) for overall CTC count, while 12/51 (23.5 \%) patients had samples with $\geq 5$ Ki67-positive CTCs. In the unfavourable prognostic category, Ki67positive CTC prevalence was higher (22/35 samples, $62.9 \%) .3 / 51$ patients (5.9 \%) had samples containing Ki67-positive CTCs exclusively, with the other 20/23 Ki67-positive CTC samples harbouring at least one Ki67-negative CTC. 307/2495 (12.3\%) of CTCs were Ki67-positive across first samples from the cohort.

\section{Prognostic impact of vimentin/Ki67 CTC expression}

We first sought to ensure that, in line with that previously described, a prognostic difference was evident across both cohorts between those patients in the unfavourable CTCpositive prognostic category $(\geq 5$ standard CTCs per $7.5 \mathrm{ml}$ ) and those in the favourable CTC-positive prognostic category $(<5$ standard CTCs per $7.5 \mathrm{ml})$ at baseline sampling.

Across the vimentin and Ki67 cohort, baseline PSA was significantly higher in those patients who had unfavourable prognosis at baseline compared to those who were in the favourable category (mean PSA 671.2 vs 278.3, $p=<0.0001$ ) (Additional file 1: Figure S2a). A significant difference in median OS was also evident in patients who were in the unfavourable category at baseline compared to those in the favourable group (375 vs 712 days,

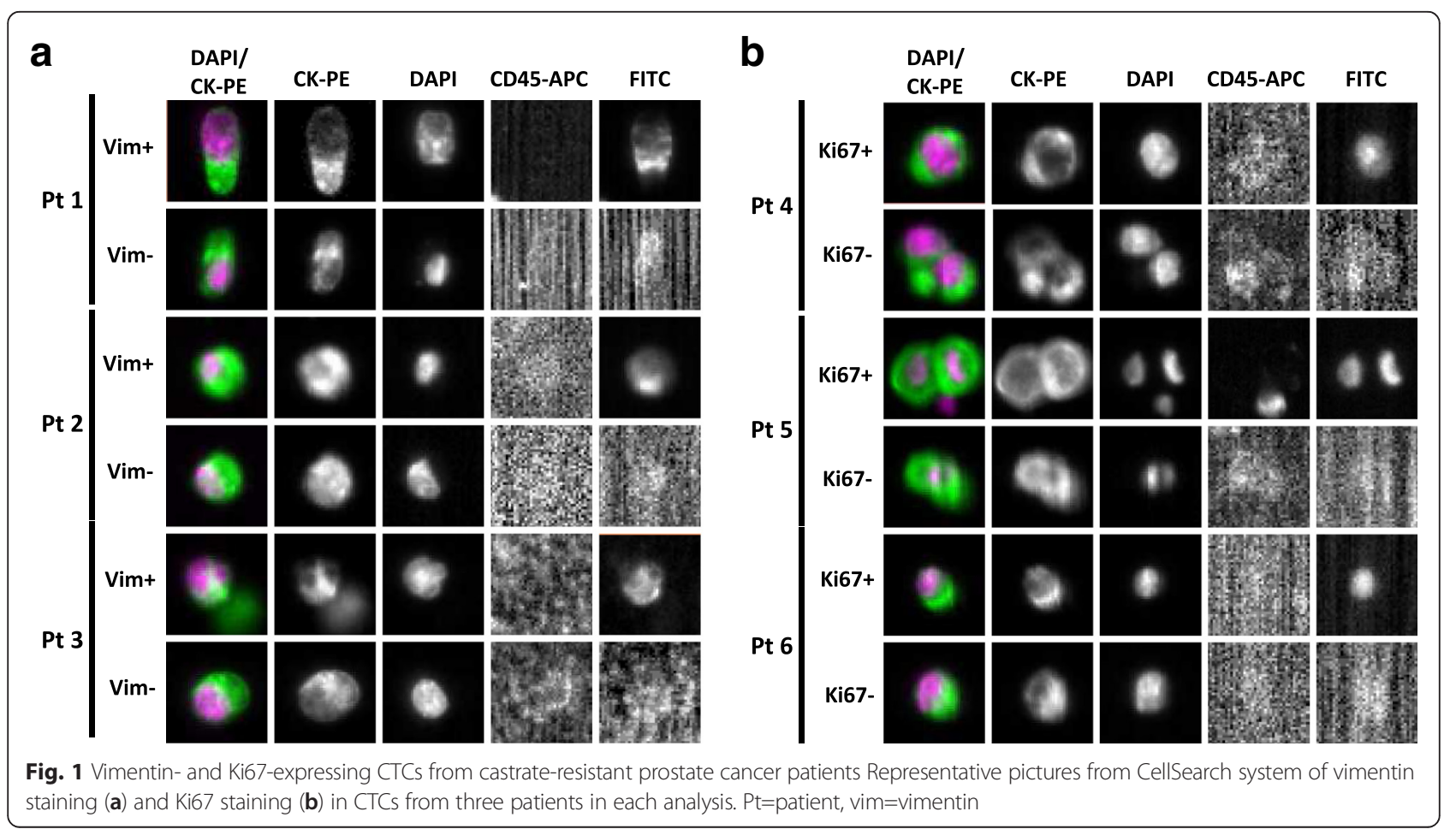


respectively; HR 2.15, $95 \%$ CI 1.35-3.04, $p=0.001$ ) (Additional file 1: Figure S2b). We thus validated that the prognostic value of standard CTCs in our two cohorts was consistent with what has been reported previously $[4,5]$.

We next asked whether the baseline presence of vimentin- or Ki67-expression in CTC could have prognostic implications. Baseline PSA was not significantly different in those patients who were CTC-vimentin positive at baseline compared to those who had no vimentin-positive CTCs (mean PSA 537.0 vs 463.2, respectively; $p=0.33$ ) (Fig. 2a). However a statistically significant difference in OS was evident between patients with vimentin-positive
CTC and those with no vimentin-positive CTC (median 305 days vs 453 days, respectively; HR 1.8, 95 \% CI 1.093.83, $p=0.0293$ ) (Fig. 2b). When sub-divided into patients belonging to the unfavourable prognostic category of $\geq 5$ standard CTCs overall, the presence of 1 vimentinpositive CTC did not diminish survival further (median 283 days vs 283 days, HR 1.33, $95 \%$ CI 0.72-2.57, $p=0.347$ ), and there was also no survival difference evident when the patients with the highest proportion of vimentin-positive CTCs were compared to those with the highest proportion of vimentin-negative CTCs (median 358 days vs 174 days, respectively; HR
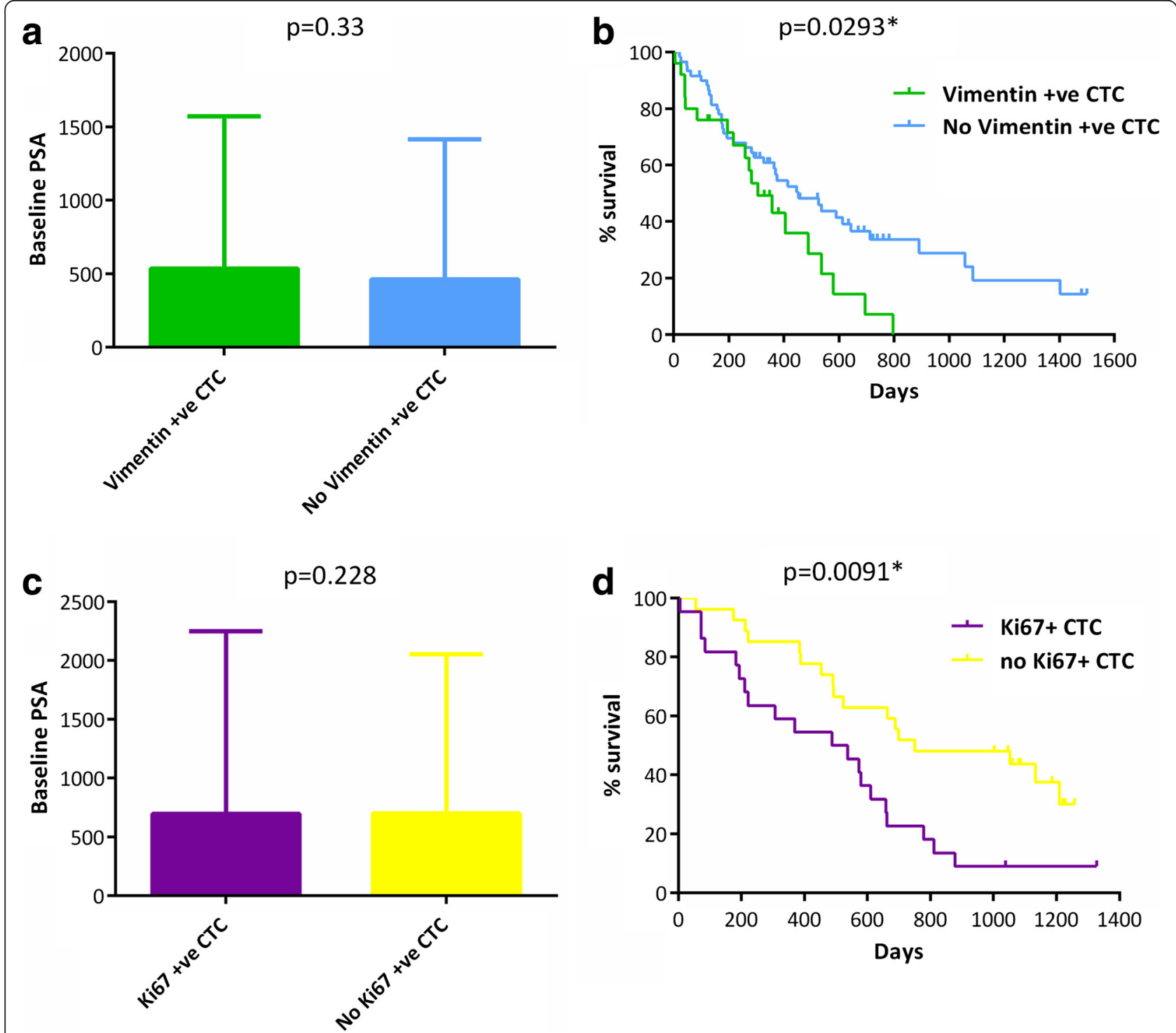

Fig. 2 Prognostic impact of vimentin- and Ki67-expressing CTC. a-b Prognostic comparison of patients with vimentin-expressing CTC at baseline compared to those with no vimentin-expressing CTC, as assessed by bar charts comparing baseline PSA (a), and Kaplan-Meier plots of overall survival (b). c-d Prognostic comparison of patients with Ki67-expressing CTC at baseline compared to those with no Ki67- expressing CTC, as assessed by bar charts comparing baseline PSA (c), and Kaplan-Meier plots of overall survival (d). Mann-Whitney tests performed for (a) and (c), log-rank tests for $(\mathbf{b})$ and $(\mathbf{d})$; Bar charts show mean $+/$ - standard deviation, $p=<0.05$ 
1.36, $95 \%$ CI $0.47-4.13, p=0.569$ ) (Additional file 1: Figure S3a, b).

In the Ki67 cohort, baseline PSA was not significantly different in those patients who were CTC Ki67-positive at baseline compared to those who had no CTC Ki67positive CTC (mean PSA 695.7 vs 697.6, respectively; $p=0.228$ ) (Fig. 2c). A statistically significant difference in OS was evident between patients with Ki67positive CTC and those with no Ki67-positive CTC (median 512 days vs 751 days, respectively; HR 2.37, $95 \%$ CI 1.35-5.23, $p=0.0091$ ), although it was not clear that the presence of 1 Ki67-positive CTC diminished survival further when patients were sub-divided into the unfavourable prognostic category of $\geq 5$ standard CTCs overall (median 537 days vs 725 days, HR 2.02, $95 \%$ CI 0.89-4.24, $p=0.099$ ) (Fig. 2d and Additional file 1: Figure S3c). There was also no survival difference evident when the patients with the highest proportion of Ki67-positive CTCs were compared to those with the highest proportion of Ki67-negative CTCs (median 600 days vs 664 days, respectively; HR 1.29, $95 \%$ CI 0.52-3.33, $p=0.581$ ) (Additional file 1: Figure S3d). Overall, this suggested that the presence of vimentin-positive or Ki67positive CTC is more clinically important than their relative numbers or proportions, and that their presence in the early stages of advanced disease could be of greater prognostic significance than in later stages.

\section{Predictive impact of vimentin/Ki67 CTC expression}

As vimentin expression has been associated with stem cell properties and treatment resistance in CTCs, we hypothesized that the mesenchymal profile of CTCs may become more apparent during treatment, even if overall CTC numbers were to diminish. We also questioned if the proliferative capacity of CTC, as represented by Ki67 expression, would be diminished by treatment, particularly those treatments with anti-proliferative properties such as docetaxel and cabazitaxel.

We first tested whether the proportion of patients with vimentin-expressing CTC had altered between baseline CTC samples and paired follow-up CTC samples taken at cycles $2-3$. When using $\geq 1$ vimentin-positive CTC as a cutoff, there was no difference in patient proportion observed: 30/93 patients harboured vimentin-expressing CTC before treatment, compared to $19 / 49$ patients after treatment ( 32.3 vs $38.8 \%, p=0.437$ ) (Table 4). There was also no difference evident using $\geq 5$ vimentin-positive CTC as a cutoff ( $10 / 93$ patients vs $7 / 49$ patients, 10.8 vs $14.3 \%$, $p=0.558$ ) (Table 4). We next asked if the proportion of vimentin-expressing CTC may change with treatment in vimentin-positive CTC patients: ratio of total CTC/vimentin-positive CTC was not significantly different before and after treatment (mean 51 at baseline vs 46.2 after treatment; $p=0.298$ ) (Fig. 3a).

For Ki67 CTC testing, no difference in patient proportion harbouring Ki67-positive CTC was observed before and after treatment, regardless of whether $\geq 1$ Ki67positive CTC or $\geq 5$ Ki67-positive CTC was used as a cutoff ( $\geq 1$ CTC: $23 / 51$ patients before vs $15 / 39$ patients after, 45.1 vs $38.5 \%, p=0.528$; $\geq 5$ CTC: $12 / 51$ patients before vs $6 / 39$ patients after, 23.5 vs $15.4 \%, p=0.336$ ) (Table 4). The proportion of Ki67-expressing CTCs relative to Ki67-negative CTC did not significantly change (mean 24.1 at baseline vs 19.3 after treatment; $p=0.85$ ) (Fig. 3b). Thus, overall there was no significant change in proportions of Ki67/vimentin patients or CTCs between baseline and post-treatment samples.

\section{Discussion}

Here we report CTC expression of vimentin and Ki67 in advanced/metastatic prostate cancer, offering a clinical insight into their mesenchymal and proliferative properties. We have established a method for testing CTC expression of Ki67 and vimentin in the normal pathway of prostate cancer care. In line with previous studies, we confirmed the prognostic value of detection of $\geq 5$ standard CTCs at baseline through analysis of PSA and OS. No significant difference was evident when baseline PSA was split into vimentin-positive and Ki67-positive CTC cohorts, but there was a significantly reduced OS in the vimentin-positive and Ki67-positive CTC cohorts compared to those patients with no vimentin/Ki67-positive CTCs, respectively. In both Ki67 and vimentin cohorts, no additional prognostic significance of Ki67- or vimentinpositive CTCs was evident when cohorts were subdivided into the unfavourable prognostic category of $\geq 5$ standard CTCs overall, or when patients with high proportions of Ki67/vimentin-positive CTCs were compared to patients with high proportions of Ki67/vimentin-negative CTCs. This suggests that the presence of vimentin-positive or Ki67-positive CTC is more clinically important than their relative numbers or proportions, and that their presence in the early stages of advanced disease could be of greater prognostic significance than in later stages. Finally, there was no change in patient percentage or CTC proportion with Ki67- or vimentin-expressing CTCs before and after treatment, suggesting the potential of these techniques to offer predictive benefits of treatment remains unestablished.

It is important to reflect on the deficiencies of this dataset and its analysis. The results here are hypothesisgenerating and should be interpreted within the context of a retrospective observational design. Survival data reported was not adjusted for patient treatment, and mCRPC patient numbers tested were relatively small, particularly for the Ki67 cohort. The variability of 
Table 4 Change in patient Ki67 and vimentin CTC profile with treatment. Chi-squared test to analyse CTC frequencies in treatment groups. Samples from the second column were taken at cycles 2-3 following initiation of treatment

\begin{tabular}{llll}
\hline & Baseline sample before treatment (\%) & First sample after treatment start (\%) & $p=0.528$ \\
\hline Number of patients with Ki67 + ve CTC (\%) & $23 / 51(45.1)$ & $15 / 39(38.5)$ & $0.39(15.4)$ \\
Number of patients with $\geq 5$ Ki67 + ve CTC (\%) & $12 / 51(23.5)$ & $19 / 49(38.8)$ & $p=0.336$ \\
Number of patients with Vim + ve CTC (\%) & $30 / 93(32.3)$ & $7 / 49(14.3)$ & $p=0.437$ \\
\hline
\end{tabular}

planned treatments was the reason progression-free survival was not evaluated in our study: with small patient numbers, an imbalance of treatment line and therapy was inevitable between our patient cohorts and would have confounded such an analysis. Finally, an initial biological selection during CellSearch should also be noted: it will only test for Ki67 and vimentin after patient CTC have been initially isolated using EPCAM-coated ferromagnetic beads, an epithelial-antibody based selection. This process is consistent across all patient cohorts analysed, but a fully representative picture of mesenchymal and proliferative CTCs may be impaired as a consequence. As an alternative to antibody-based CTC selection via CellSearch, a number of other CTC detection techniques are in different stages of development, some with the aim of isolating CTC by their large size rather than their biological phenotype: a similar analysis of CTC vimentin expression in $\mathrm{mCRPC}$ using these techniques would be of future interest, and could offer a more accurate picture of its clinical relevance.

We noted a significant reduction of OS in the patient cohort harbouring vimentin-expressing CTC: HR was
1.8 , with a median of 305 days OS in the vimentinpositive CTC population compared to 453 days for those patients with no vimentin-positive CTCs. Given some of the study limitations noted in the previous paragraph (ie the possibility that a high percentage of mesenchymal CTC are missed by the CellSearch technology), this finding merits further examination and has future potential to offer important insight regarding mesenchymal CTC and their treatment. It is possible that this difference could have been more profound using CTC enrichment platforms that are not antibody-based. More opportunity to perform detailed study of vimentin co-localisation with other CTC markers should hopefully arise soon, given the recent identification and clinical characterisation of a noncommercial cell surface vimentin antibody that detects CTCs from epithelial cancers with high sensitivity and specificity [28].

Mesenchymal or EMT CTC, as represented by vimentin expression in this study, have previously been associated with tumour invasiveness, chemoresistance, CTC clustering, stem cell characteristics, and poorer clinical outcomes $[29,30]$. Although not validated by our study, the results
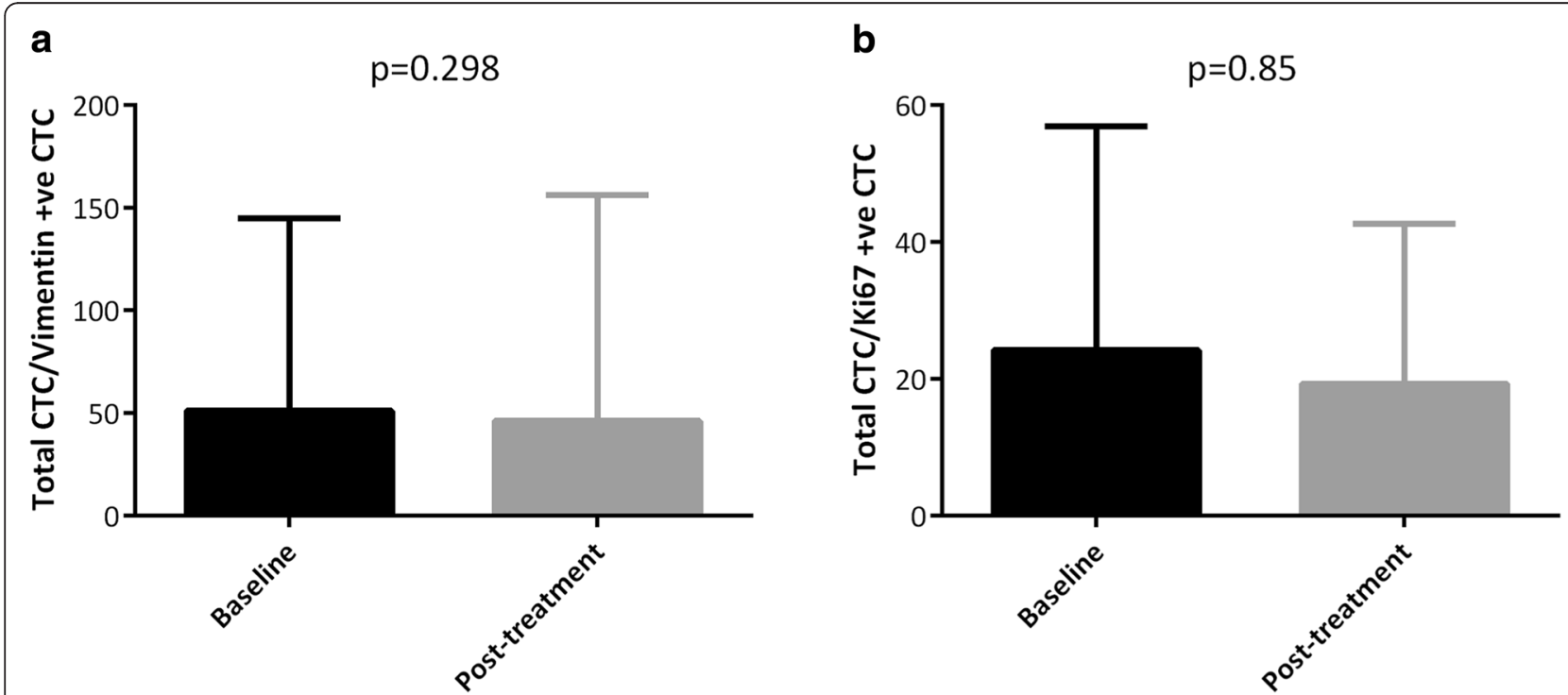

Fig. 3 Change in vimentin- and Ki67-expressing CTC after initiation of treatment a) Bar chart assessing proportion of vimentin-positive CTC expression, compared to total CTC expression, at baseline and after treatment start b) Bar chart assessing proportion of Ki67-positive CTC expression, compared to total CTC expression, at baseline and after treatment start. 'Post-treatment' samples were taken at cycles 2-3 following initiation of treatment. Mann-Whitney test; Bar charts show mean + /- standard deviation, $p=<0.05$ 
generated here are generally consistent with this proposed biological and clinical phenotype. In parallel with the poorer prognosis seen in this study for those patients with vimentin-positive CTCs at baseline, the lack of survival difference observed in those patients whose samples contained the highest proportion of vimentin-positive CTCs is consistent with (but does not confirm) the stem cell theory that just one vimentin-positive cell will suffice to contribute to poorer outcomes. Armstrong and colleagues previously used the CellSearch platform to offer a detailed biological examination of vimentin and other markers of the EMT program in samples from patients with breast and prostate cancer, revealing the existence and high frequency of CTCs that can co-express epithelial and mesenchymal proteins [25]. Using a cutoff of 1 vimentinpositive CTC, 30/93 CRPC patients from our study had baseline samples with CTCs that co-expressed cytokeratin with vimentin, compared to 10/10 CRPC patients documented previously. This disparity could be accounted for by differences between patient characteristics and staining methods employed in the two studies, but here we have also been able to build on the clinical aspect of vimentin analysis by offering the larger clinical cohort with longer-term follow-up that was recommended in the previous study.

A significant reduction of OS was also observed in the patient cohort harbouring Ki67-expressing CTC: HR was 2.37, with a median of 512 days OS in the Ki67-positive CTC population compared to 751 days for those patients with no Ki67-positive CTCs. One previous study has explored the biological character of Ki67-positive CTC using an alternative CTC isolation platform, also offering some preliminary insight from CTC enumeration of 10 patient samples: $12.3 \%$ of CTCs were Ki67-positive in our study, compared to 7-73\% from 4 CRPC patients reported previously [18]. We have thus been able to consolidate the results of this initial study using larger patient numbers and the CellSearch platform, suggesting again that there is potential prognostic clinical insight to be gained by examining proliferative and non-proliferative CTC subpopulations. Future studies can now focus prospectively on prognostic validation of Ki67-positive CTCs and their response to particular treatment types.

A number of other prostate cancer CTC studies have recently elaborated on the original work which showed the prognostic value of CTC in mCRPC $[4,5]$. In terms of CTC enumeration, a putative predictive role for 'categorical' CTC enumeration (ie whether a patient is CTC positive or not) has been suggested after 1 cycle of chemotherapy, an evaluation that can be performed weeks ahead of standard response approaches such as PSA and CT [31]. A recent large prospective study suggested that CTC count and LDH value could be a surrogate of overall survival in mCRPC treated with abiraterone [6]. On the other end of the research spectrum, the biological heterogeneity of prostate cancer with treatment has also recently been re-illustrated despite its seemingly predictable clinical pattern of metastatic spread compared to many other cancers: CTC measurement and next generation single cell sequencing of metastatic prostate cancer patient revealed a vast complexity of clonal evolution, a finding consistent with the metastasis-to-metastasis spread of multiple tumour clones recently revealed by prostate tumour biopsies [32-34]. Many of these studies have concentrated on androgen receptor expression and its predictive capacity $[11,12,35]$. Overall, the vast range of CTC enumeration, genomic and biological progress achieved in these studies offer an encouraging window of insight towards the future for circulating biomarkers in prostate cancer.

\section{Conclusions}

Here we show that routine clinical testing of vimentin and Ki67 expression in CTC derived from mCRPC patients is feasible. A significant reduction in OS for patients with baseline CTC vimentin and Ki67 expression suggests that largescale prospective analyses would be important to further explore these potential links. Future studies will also require further detailed correlation of vimentin/Ki67 CTC expression and response to certain treatment types: for example, the predictive capacity of lost or increased Ki67 CTC-expression in a large cohort of mCRPC patients treated exclusively with anti-proliferative chemotherapy agents such as docetaxel or cabazitaxel. Finally, a number of alternative CTC-isolation techniques have been developed and merit further analysis for such clinical correlation studies: size-based filtration techniques may be particularly helpful for vimentin-expressing CTC which may not be efficiently captured by techniques based on epithelial-antibody expression.

\section{Additional file}

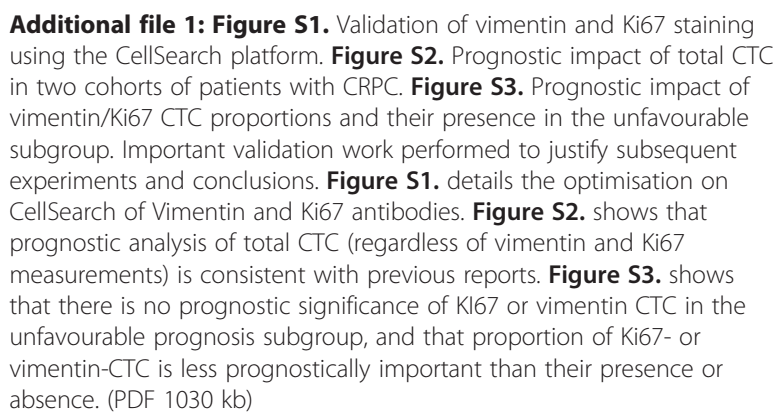

\section{Abbreviations}

AR: androgen receptor; CRPCcastrate-resistant: prostate cancer; CTCcirculating: tumor cell; EMTepithelial-mesenchymal: transition; HR: hazard ratio; IF: immuno-fluorescence; mCRPC: metastatic castrateresistant prostate cancer; OS: overall survival; PSA: prostate-specific antigen; RTOG: Radiation Therapy Oncology Group. 


\section{Competing interests}

The authors declare they have no competing interests.

\section{Authors' contributions}

Conception and design of the study was performed by CRL, SLM, YL, PV, KF, CM and FF; acquisition of data was performed by CRL, SLM, FB, YL, MN, PV, $\mathrm{KF}, \mathrm{CM}$ and FF; interpretation and analysis of data by CRL with review and comment from SLM, FB, YL, MN, PV, KF, CM and FF. Manuscript first drafted by FF and CRL, with contributions for final draft from SLM, FB, YL, MN, PV, KF and CM. All authors read and approved the final manuscript.

\section{Acknowledgements}

Thank you to the Institut National du Cancer and Cancéropôle lle de France for their funding of the PAIR prostate program contributing to this research (Grant No 2010-174). CL was supported by ESMO [translational research fellowship] with the aid of a grant from Hoffmann-La Roche. Any views, opinions, findings, conclusions, or recommendations expressed in this material are those solely of the author(s) and do not necessarily reflect those of ESMO or Hoffmann-La Roche. CL is also a recipient for the grant DUERTECC/EURONCO (Diplôme Universitaire Européen de Recherche Translationelle et Clinique en Cancérologie)

\section{Author details}

'INSERM U981, University of Paris-Sud XI, Translational Research Laboratory, Gustave Roussy Cancer Campus, Villejuif, France. ${ }^{2}$ Department of Medical Oncology, Bergonie Cancer Institute, Bordeaux, France. ${ }^{3}$ Department of Medical Oncology, Gustave Roussy Cancer Campus, Villejuif, France. ${ }^{4}$ Department of Biopathology, Gustave Roussy Cancer Campus, Villejuif, France.

\section{Received: 20 October 2015 Accepted: 17 February 2016}

\section{Published online: 29 February 2016}

\section{References}

1. Fizazi K, Morat L, Chauveinc L, Prapotnich D, De Crevoisier R, Escudier B, et al. High detection rate of circulating tumor cells in blood of patients with prostate cancer using telomerase activity. Ann Oncol. 2007:18(3):518-21.

2. Armstrong AJ, Eisenberger MA, Halabi S, Oudard S, Nanus DM, Petrylak DP, et al. Biomarkers in the management and treatment of men with metastatic castration-resistant prostate cancer. Eur Urol. 2012;61(3):549-59.

3. Miyamoto DT, Sequist LV, Lee RJ. Circulating tumour cells-monitoring treatment response in prostate cancer. Nat Rev Clin Oncol. 2014;11(7):401-12.

4. de Bono JS, Scher HI, Montgomery RB, Parker C, Miller MC, Tissing H, et al. Circulating tumor cells predict survival benefit from treatment in metastatic castration-resistant prostate cancer. Clin Cancer Res. 2008;14(19):6302-9.

5. Olmos D, Arkenau HT, Ang JE, Ledaki I, Attard G, Carden CP, et al. Circulating tumour cell (CTC) counts as intermediate end points in castration-resistant prostate cancer (CRPC): a single-centre experience. Ann Oncol. 2009;20(1):27-33.

6. Scher HI, Heller G, Molina A, Attard G, Danila DC, Jia X, et al. Circulating tumor cell biomarker panel as an individual-level surrogate for survival in metastatic castration-resistant prostate cancer. J Clin Oncol. 2015;33(12):1348-55.

7. Maheswaran S, Sequist LV, Nagrath S, Ulkus L, Brannigan B, Collura CV, et al. Detection of mutations in EGFR in circulating lung-cancer cells. N Engl J Med. 2008;359(4):366-77.

8. Pailler E, Adam J, Barthélémy A, Oulhen M, Auger N, Valent A, et al. Detection of circulating tumor cells harboring a unique ALK rearrangement in ALK-positive non-small-cell lung cancer. J Clin Oncol. 2013;31(18):2273-81.

9. Pailler E, Auger N, Lindsay CR, Vielh P, Islas-Morris-Hernandez A, Borget et al. High level of chromosomal instability in circulating tumor cells of ROS1-rearranged non-small-cell lung cancer. Ann Oncol. 2015;26(7):1408-15.

10. Attard G, de Bono JS, Logothetis CJ, Fizazi K, Mukherjee SD, Joshua AM, et al. Improvements in radiographic progression-free survival stratified by ERG gene status in metastatic castration-resistant prostate cancer patients treated with abiraterone acetate. Clin Cancer Res. 2015;21(7):1621-7.

11. Nakazawa M, Lu C, Chen Y, Paller CJ, Carducci MA, Eisenberger MA, et al. Serial blood-based analysis of ar-v7 in men with advanced prostate cancer. Ann Oncol. 2015;26(9):1859-65.

12. Crespo M, van Dalum G, Ferraldeschi R, et al. Androgen receptor expression in circulating tumour cells from castration-resistant prostate cancer patients treated with novel endocrine agents. Br J Cancer. 2015;112(7):1166-74.
13. Pollack A, DeSilvio M, Khor LY, Li R, Al-Saleem TI, Hammond ME, et al. Ki-67 staining is a strong predictor of distant metastasis and mortality for men with prostate cancer treated with radiotherapy plus androgen deprivation: Radiation Therapy Oncology Group Trial 92-02. J Clin Oncol. 2004;22(11):2133-40

14. Khor LY, Bae K, Paulus R, Al-Saleem T, Hammond ME, Grignon DJ, et al. MDM2 and Ki-67 predict for distant metastasis and mortality in men treated with radiotherapy and androgen deprivation for prostate cancer: RTOG 92-02. J Clin Oncol. 2009;27(19):3177-84.

15. Fisher G, Yang ZH, Kudahetti S, Møller H, Scardino P, Cuzick J, et al. Prognostic value of $\mathrm{Ki}-67$ for prostate cancer death in a conservatively managed cohort. Br J Cancer. 2013;108(2):271-7.

16. Tollefson MK, Karnes RJ, Kwon ED, Lohse CM, Rangel LJ, Mynderse LA, et al. Prostate cancer Ki-67 (MIB-1) expression, perineural invasion, and gleason score as biopsy-based predictors of prostate cancer mortality: the Mayo model. Mayo Clin Proc. 2014;89(3):308-18

17. Rajpar S, Vielh P, Laplanche A, Lesaunier F, Delva R, Gravis G, et al. A study of ERG, PTEN, and ki-67 in a phase III trial assessing docetaxel and estramustine in high-risk localized prostate cancer (GETUG 12). J Clin Oncol. 2014;32:5s (abstr 5063).

18. Stott SL, Lee RJ, Nagrath S, Yu M, Miyamoto DT, Ulkus L, et al. Isolation and characterization of circulating tumor cells from patients with localized and metastatic prostate cancer. Sci Transl Med. 2010;2(25):25ra23.

19. Thiery JP, Acloque H, Huang RY, Nieto MA. Epithelial-mesenchymal transitions in development and disease. Cell. 2009;139(5):871-90.

20. Kalluri R, Weinberg RA. The basics of epithelial-mesenchymal transition. J Clin Invest. 2009;119(6):1420-8.

21. Farace $F$, Massard $C$, Vimond N, Drusch F, Jacques N, Billiot F, et al. A direct comparison of Cell Search and ISET for circulating tumour-cell detection in patients with metastatic carcinomas. Br J Cancer. 2011;105(6):847-53.

22. Hofman V, Ilie MI, Long E, Selva E, Bonnetaud C, Molina T, et al. Detection of circulating tumor cells as a prognostic factor in patients undergoing radical surgery for non-small-cell lung carcinoma: comparison of the efficacy of the Cell Search Assay ${ }^{\mathrm{TM}}$ and the isolation by size of epithelial tumor cell method. Int J Cancer. 2011;129(7):1651-60.

23. Lecharpentier A, Vielh P, Perez-Moreno P, Planchard D, Soria JC, Farace F. Detection of circulating tumour cells with a hybrid (epithelial/mesenchymal) phenotype in patients with metastatic non-small cell lung cancer. $\mathrm{Br}$ J Cancer. 2011;105(9):1338-41.

24. Krebs MG, Hou JM, Sloane R, Lancashire L, Priest L, Nonaka D, et al. Analysis of circulating tumor cells in patients with non-small cell lung cancer using epithelial marker-dependent and -independent approaches. J Thorac Oncol. 2012;7(2):306-15.

25. Armstrong AJ, Marengo MS, Oltean S, Kemeny G, Bitting RL, Turnbull JD, et al. Circulating tumor cells from patients with advanced prostate and breast cancer display both epithelial and mesenchymal markers. Mol Cancer Res. 2011;9(8):997-1007

26. Cristofanilli M, Budd GT, Ellis MJ, Stopeck A, Matera J, Miller MC, et al. Circulating tumor cells, disease progression, and survival in metastatic breast cancer. N Engl J Med. 2004;351(8):781-91.

27. Allard WJ, Matera J, Miller MC, Repollet M, Connelly MC, Rao C, et al. Tumor cells circulate in the peripheral blood of all major carcinomas but not in healthy subjects or patients with nonmalignant diseases. Clin Cancer Res. 2004;10(20):6897-904

28. Satelli A, Mitra A, Brownlee Z, Xia X, Bellister S, Overman MJ, et al. Epithelialmesenchymal transitioned circulating tumor cells capture for detecting tumor progression. Clin Cancer Res. 2015;21(4):899-906.

29. Wu S, Liu S, Liu Z, Huang J, Pu X, Li J, et al. Classification of circulating tumor cells by epithelial-mesenchymal transition markers. PLoS One. 2015;10(4):e0123976

30. Krebs MG, Metcalf RL, Carter L, Brady G, Blackhall FH, Dive C. Molecular analysis of circulating tumour cells-biology and biomarkers. Nat Rev Clin Oncol. 2014:11(3):129-44

31. Thalgott M, Rack B, Eiber M, Souvatzoglou M, Heck MM, Kronester C, et al. Categorical versus continuous circulating tumor cell enumeration as early surrogate marker for therapy response and prognosis during docetaxel therapy in metastatic prostate cancer patients. BMC Cancer. 2015:15:458

32. Hong MK, Macintyre G, Wedge DC, Van Loo P, Patel K, Lunke S, et al. Tracking the origins and drivers of subclonal metastatic expansion in prostate cancer. Nat Commun. 2015;6:6605. 
33. Gundem G, Van Loo P, Kremeyer B, Alexandrov LB, Tubio JM, Papaemmanuil E, et al. The evolutionary history of lethal metastatic prostate cancer. Nature. 2015;520(7547):353-7.

34. Robinson D, Van Allen EM, Wu YM, Schultz N, Lonigro RJ, Mosquera JM, et al. Integrative clinical genomics of advanced prostate cancer. Cell. 2015;161(5):1215-28.

35. Dago AE, Stepansky A, Carlsson A, Luttgen M, Kendall J, Baslan T, et al. Rapid phenotypic and genomic change in response to therapeutic pressure in prostate cancer inferred by high content analysis of single circulating tumor cells. PLoS One. 2014;9(8):e101777.

Submit your next manuscript to BioMed Central and we will help you at every step:

- We accept pre-submission inquiries

- Our selector tool helps you to find the most relevant journal

- We provide round the clock customer support

- Convenient online submission

- Thorough peer review

- Inclusion in PubMed and all major indexing services

- Maximum visibility for your research

Submit your manuscript at www.biomedcentral.com/submit 